\section{Benefícios auxílio-doença concedidos aos trabalhadores empregados no ramo de carne e pescado no Brasil em 2008}

\author{
Sick leave benefits for workers in the Brazilian \\ meat and fish industries in 2008
}

Prestaciones sociales por enfermedad, concedidas
por el Seguro Social, a los empleados en el
sector de la carne y pescado en Brasil
durante el año 2008

\begin{abstract}
${ }_{1}$ Universidade de Brasília Brasília, Brasil.

2 Faculdades São Lucas, Porto Velho, Brasil.

3 Universidade Federal de

Rondônia, Porto Velho, Brasil.

4 Fundação Osvaldo Cruz,

Porto Velho, Brasil.

5 Universidade de São Paulo,

São Paulo, Brasil.

Correspondência

H. R. Jakobi

Rua Thales Benevides 5355

apto. 1003, Porto Velho, RO

76821-348, Brasil.

heinzjakobi@gmail.com
\end{abstract}

\begin{abstract}
This study aims to analyze factors associated with sick leave rates among workers in the meat, fish, and seafood industries in Brazil. The study analyzed all sick leave benefits granted by the country's social security system to workers in these industries in 2008. Incidence of sick leave per 10 thousand jobs was stratified by sex, age, diagnosis, job position, State, and nature and length of benefits. The study analyzed 31,913 sick leaves, with an annual incidence of 788.7. Meat processing and packaging showed the highest incidence, and fish and seafood processing and packaging showed the longest mean length of sick leave. Women showed a higher sick leave incidence, while men received longer average sick leaves. Injuries, musculoskeletal disorders, and mental disorders accounted for $67.2 \%$ of sick leaves. The most common diagnoses were lower back pain, first-trimester bleeding in pregnancy, and depression. The data suggest poor job protection and adverse working conditions in these industries.
\end{abstract}

Social Security; Salaries and Fringe Benefits; Occupational Health; Abattoirs; Fishing Industry
Heinz Roland Jakobi 1,2

Anadergh Barbosa-Branco 1

Luis Fernando Bueno 3

Ricardo de Godoi Mattos Ferreira 4 Luís Marcelo Aranha Camargo 5

\section{Resumo}

Este estudo objetivou identificar fatores associados à incidência de concessão de benefícios entre trabalhadores na atividade de carne e pescado no Brasil. Foram estudados os benefícios auxíliodoença concedidos pela Previdência Social aos empregados em carne e pescado no Brasil em 2008. As incidências por 10 mil vínculos empregatícios foram estratificadas por sexo, idade, diagnóstico, atividade econômica, unidade federada, espécie e duração dos benefícios auxílio-doença. Foram concedidos 31.913 benefícios auxílio-doença, com incidência de 788,7. O ramo abate de bovinos, equinos, ovinos e caprinos apresentou a maior incidência (1.223,2); o pescado, a maior duração (61 dias) de benefícios auxílio-doença. As mulheres apresentaram maior incidência, enquanto os homens, maior duração de benefícios auxílio-doença. Os grupos Lesões, doenças osteomusculares e transtornos mentais representaram $67,2 \%$ dos benefícios auxílio-doença. Os diagnósticos mais incidentes foram as dorsopatias, hemorragia no início da gravidez e episódios depressivos. O ramo de atividade, sexo, e a idade representaram importantes fatores associados à incidência de benefícios auxílio-doença, sugerindo uma precarização das condições/relações de trabalho.

Previdência Social; Salários e Benefícios; Saúde do Trabalhor; Matadouros; Industria Pesqueira 


\section{Introdução}

A indústria frigorífica faz parte da indústria de transformação e representa um sistema contínuo de "desmontagem" do animal, o que torna o trabalho exaustivo e perigoso ${ }^{1}$. Nessa atividade existem fortes indícios de precarização do emprego e das condições de trabalho 2 .

As condições de trabalho constituem importante fator para a incapacidade para o trabalho 3 . A maioria das empresas frigoríficas possuem fatores de risco que devem ser considerados no contexto da saúde do trabalhador e da incapacidade para o trabalho, como as características do processo produtivo, a organização de trabalho, as características socioeconômicas e educacionais, os sistemas de compensações e gratificações e as relações de poder entre empregador/ empregado ${ }^{4}$.

Vários são os fatores de risco presentes nos ambientes de trabalho em frigoríficos, com destaque para agentes físicos (ruído, temperaturas extremas, umidade, vibração), biológicos, químicos e ergonômicos (força, repetitividade, posturas estereotipadas, linha de produção). Associados a esses existem fatores psicossociais relacionados tanto às características das condições de produção (elevada demanda de trabalho, longas jornadas, dessincronismo do ritmo biológico, baixa mecanização, equipamento de trabalho inadequado, baixa escolaridade, baixos salários), quanto à percepção de sofrimento no trabalho representado pela matança de animais, muitas vezes de formas cruéis, odor desagradável etc. Tais fatores psicossociais contribuem para o aumento da tensão psicológica, violência e angústia, traduzidos como sofrimento no trabalho 5,6.

Recentes estudos brasileiros sobre incapacidade para o trabalho no Brasil apontaram o ramo econômico fabricação de produtos alimentícios como o sexto na incidência de incapacidade para o trabalho (657,3/10 mil vínculos empregatícios) independentemente do grupo diagnóstico e da espécie de benefício; e quinto em acidentes do trabalho 3,7,8. Quando considerado por grupos diagnósticos específicos como as doenças osteomusculares $(176,9) 8$ e os transtornos mentais $(62,6)^{3}$, nesse ranking, ficou em 10o e 12o lugares, respectivamente.

Os benefícios auxílio-doença constituem-se num bom indicador das condições de saúde da população trabalhadora segurada, particularmente daqueles que resultam em condição clínica mais severa com afastamento maior de 15 dias e num maior risco para aposentadoria por invalidez e mortalidade ?

Este estudo objetivou identificar fatores associados à incidência de concessão de benefí- cios entre trabalhadores na atividade de carne e pescado no Brasil.

\section{Métodos}

Trata-se de um estudo epidemiológico de base populacional, baseado nos benefícios auxílio-doença concedidos pelo Instituto Nacional de Seguridade Social (INSS) aos trabalhadores empregados no ramo de atividade econômica da indústria de transformação na fabricação de produtos alimentícios no sub-ramo de abate e fabricação de produtos de carne e da preservação do pescado e fabricação de produtos do pescado (carne e pescado), no Brasil, em 2008.

A população do estudo compreendeu todos os trabalhadores empregados nas atividades de carne e pescado, obtida pela média mensal dos registros de emprego declarados pelos empregadores ao Cadastro Nacional de Informações Sociais (CNIS), em 2008. Maiores detalhes sobre o cálculo da população e o processo de concessão dos benefícios auxílio-doença encontram-se descritos em outros estudos 3,7,8,9.

Os dados dos benefícios são provenientes do Sistema Único de Benefícios do INSS e compreenderam todos os benefícios auxílio-doença concedidos aos trabalhadores dos seguintes sub-ramos (Cadastro Nacional de Atividades Econômicas - CNAE-classe 10): 1011 - abate de bovinos, equinos, ovinos e caprinos - frigoríficos; 1012 - abate de suínos, aves e pequenos animais - frigoríficos; 1013 - fabricação de produtos de carne e preparo de subprodutos do abate; e 1020 - preservação do pescado e fabricação de produtos de pescado, iniciados no período de lo de janeiro a 31 de dezembro de 2008.

Os dados foram tabulados e analisados no programa Microsoft Office Excel (Microsoft Corp., Estados Unidos) e no IBM SPSS Statistics Standard versão 19 (IBM Corp., Armonk, Estados Unidos). A taxa de incidência (por 10 mil vínculos empregatícios) foi calculada pela soma dos benefícios auxílio-doença, dividida pela média mensal dos vínculos empregatícios em 2008.

A incidência de benefícios foi estratificada por sexo (masculino e feminino); idade (faixas etárias < 20; 20-29; 30-39; 40-49; 50-59; $\geq 60$ anos); Classificação Internacional de Doenças - 10a revisão (CID-10) (grupo, agrupamento e categoria diagnóstica); atividade econômica (CNAE-classe); Unidade Federativa (UF); e espécie de benefício (auxílio-doença previdenciário, auxíliodoença acidentário). A duração do benefício foi calculada por meio da curva de sobrevivência do benefício (Kaplan-Meier) e de medida de tendência central (mediana). A análise da duração 
dos benefícios auxílio-doença utilizou metodologia não paramétrica, devido à ausência de distribuição normal.

As distorções geradas por valores numéricos (n) muito pequenos, tanto para o CNIS (denominador) quanto para os benefícios (numerador), representam um viés de cálculo importante. Antes de fazer o ranking das prevalências, foram separadas da análise individual as CNAE-classe com CNIS menor que 500 trabalhadores e/ou menos de 20 benefícios, critérios esses independentes, definidos como parâmetros de corte. Esses dados foram agrupados na categoria "outros", consolidando o total de benefícios. Os resultados agrupados em "outros" não foram considerados na análise individualizada das categorias.

\section{Aspectos éticos}

Este projeto de pesquisa foi aprovado pelo Parecer Consubstanciado no 135.115 do Comitê de Ética em Pesquisa (CEP) das Faculdades Integradas Aparício Carvalho, Porto Velho, Rondônia em 30/Out/2012.

\section{Resultados}

Em 2008, o INSS concedeu 31.913 benefícios auxílio-doença aos 404.636 trabalhadores das atividades de carne e pescado, que resultou em incidência de 788,7 benefícios/10 mil vínculos empregatícios. A incidência de benefícios previdenciários foi de 537,0 e a de benefícios acidentários foi de 251,7.

As variações de incidências entre o conjunto das unidades federativas foram de até $250 \%$; as unidades da Região Sul do Brasil apresentaram as maiores taxas (Tabela 1).

Quanto à CNAE-classe, observou-se (Tabela 1) grande variabilidade nas taxas de incidência de benefícios auxílio-doença, variando de 411,8 na fabricação de produtos de carne e preparo de subprodutos do abate (CNAE 1013) a 1.223,2 no abate de bovinos, equinos, ovinos e caprinos frigoríficos (CNAE 1011). A incidência de BAD entre os trabalhadores da CNAE 1011 foi 55\% superior à média do conjunto carne e pescado e 197\% superior a do CNAE 1013 (fabricação de produtos de carne e preparo de subprodutos do abate).

Ao analisar esse indicador em relação ao CNAE-classe e à unidade da federação, encontrou-se maior incidência de benefícios auxílio-doença entre os trabalhadores de abate de bovinos, equinos, ovinos e caprinos - frigoríficos nos estados das regiões Sul e Sudeste, exceto no Espírito Santo e Rio de Janeiro, uma vez que nesses estados predominaram os benefícios auxílio-doença no ramo de pescado. O estado do Maranhão destacou-se por apresentar maiores incidências de benefícios auxílio-doença nos ramos de abate de suínos, aves e pequenos animais e no de fabricação de produtos de carne e preparo de subprodutos do abate.

A CNAE 1011 apresentou a maior incidência de benefícios, independentemente do diagnóstico, espécie de benefício, idade e de sexo. Essa CNAE, quando analisado segundo sexo e idade, mostrou maior incidência entre as mulheres (64\%) do que entre os homens, com aumento progressivo com a idade. A maior caracterização de nexo técnico (relação com o trabalho) segundo a idade e a atividade econômica foi verificada na faixa etária menor que 20 anos, independente da CNAE-classe (Tabela 2).

A razão de incidência entre as espécies de benefícios nas diversas CNAE-classe apresentou discreta variação (máximo de 20\%), independentemente do sexo, com predomínio de benefícios auxílio-doença previdenciário.

Esses dados mostram que os homens caracterizam mais agravos como relacionados com o trabalho do que as mulheres, exceto no ramo pescado (Tabela 3).

Ao analisar a incidência de benefícios auxílio-doença segundo os grupos (capítulos) da CID-10, observou-se que $67,2 \%$ dos benefícios foram em razão de apenas três grupos da CID10: lesões; doenças do sistema osteomuscular e do tecido conjuntivo; e transtornos mentais e comportamentais. Diferentes rankings de incidência para os grupos CID-10 foram encontrados para homens e mulheres, tanto para benefícios previdenciários quanto acidentários. Entre os benefícios previdenciários (B31), houve maior predomínio de lesões entre os homens e de doenças mentais entre mulheres, enquanto entre os benefícios acidentários (B91) as Lesões também predominaram entre os homens e as doenças osteomusculares e do tecido conjuntivo entre as mulheres. Ao considerar apenas benefícios auxílio-doença acidentários, as taxas foram mais elevadas entre as mulheres do que entre os homens (Tabela 4).

Chama a atenção a elevada razão de feminilidade $(6,3: 1,0)$ observada entre os sexos para o grupo das neoplasias. Nesse grupo, os benefícios auxílio-doença ficaram restritos aos benefícios previdenciários (Tabela 4).

A Tabela 5 apresenta a incidência de benefícios auxílio-doença segundo a categoria diagnóstica da CID-10 e mostra a preponderância das hemorragias do início da gravidez $(55,3)$ das dorsopatias $(50,8)$ e dos episódios depressivos $(44,8)$, no entanto, se forem considerados os episódios 
Prevalência * de benefícios auxílio-doença segundo atividade econômica Cadastro Nacional de Atividades Econômicas (CNAE-classe) e Unidade Federativa (UF) no sub-ramo carne e pescado. Brasil, 2008.

\begin{tabular}{|c|c|c|c|c|c|c|}
\hline UF & $\mathbf{n}$ & 1011 & 1012 & 1013 & 1020 & Total \\
\hline Rio Grande do Sul & 6.102 & $2.308,1$ & $1.151,3$ & $1.180,3$ & $1.079,3$ & $1.313,5$ \\
\hline Minas Gerais & 2.866 & $1.560,2$ & 448,3 & 602,1 & 147,1 & 858,2 \\
\hline Santa Catarina & 5.347 & $5.794,4$ & 611,1 & 552,7 & 995,1 & 817,6 \\
\hline Mato Grosso do Sul & 1.691 & 857,7 & 553,3 & 502,4 & 828,6 & 741,3 \\
\hline Paraná & 4.694 & $1.606,2$ & 649,5 & 401,9 & 734,8 & 726,5 \\
\hline São Paulo & 4.441 & $1.573,7$ & 643,4 & 244,7 & 379,1 & 676,4 \\
\hline Rio de Janeiro & 628 & 793,0 & 783,4 & 436,7 & $1.263,4$ & 668,7 \\
\hline Mato Grosso & 1.852 & 757,8 & 360,5 & 517,2 & $1.179,4$ & 663,7 \\
\hline Goiás & 1.741 & $1.101,5$ & 229,9 & 193,3 & 0,0 & 631,7 \\
\hline Sergipe & 24 & $1.155,0$ & 168,6 & 178,6 & $1.043,5$ & 588,2 \\
\hline Rondônia & 442 & 582,5 & 369,0 & 868,5 & 475,2 & 576,5 \\
\hline Espírito Santo & 170 & 907,9 & 173,1 & 267,3 & $2.033,9$ & 567,0 \\
\hline Ceará & 57 & 629,9 & 455,4 & 341,8 & 786,8 & 539,0 \\
\hline Piauí & 13 & $1.024,2$ & 351,5 & 0,0 & $1.111,1$ & 519,0 \\
\hline Maranhão & 86 & 452,1 & $3.564,4$ & $2.264,2$ & $1.600,0$ & 479,2 \\
\hline Acre & 41 & 467,3 & 0,0 & 0,0 & 0,0 & 448,5 \\
\hline Paraíba & 11 & $1.524,5$ & 150,8 & 244,4 & 310,9 & 437,2 \\
\hline Tocantins & 166 & 433,4 & 439,3 & 590,5 & 0,0 & 435,1 \\
\hline Pernambuco & 172 & 851,9 & 308,8 & 441,5 & 458,4 & 387,9 \\
\hline Pará & 308 & 380,7 & 213,4 & 302,8 & 489,6 & 386,9 \\
\hline Bahia & 174 & 697,0 & 236,0 & 68,8 & 207,0 & 376,2 \\
\hline Rio Grande do Norte & 22 & 233,7 & $1.124,1$ & 69,9 & 523,3 & 367,7 \\
\hline Alagoas & 25 & 922,7 & 369,8 & 0,0 & 133,2 & 360,3 \\
\hline Distrito Federal & 115 & $1.036,1$ & 329,4 & 301,1 & 0,0 & 358,8 \\
\hline Amazonas & 18 & 287,0 & 0,0 & 0,0 & 167,3 & 260,0 \\
\hline Amapá & 1 & 0,0 & 0,0 & 0,0 & $5.217,4$ & 246,4 \\
\hline Total & 31.913 & $1.223,2$ & 658,7 & 411,8 & 810,2 & 788,7 \\
\hline
\end{tabular}

* Por 10 mil trabalhadores.

1011: abate de bovinos, equinos, ovinos e caprinos - frigoríficos; 1012: abate de suínos, aves e pequenos animais -

frigoríficos; 1013: fabricação de produtos de carne e preparo de subprodutos do abate; 1020: pescado - preservação

e fabricação de produtos de pescado.

depressivos (F32) e os transtornos depressivos recorrentes (F33), constata-se que a "depressão" representa a principal categoria diagnóstica, totalizando uma incidência de 57,1/10 mil vínculos empregatícios. Quanto às espécies de benefícios, observou-se que entre os previdenciários se destacaram as mesmas três categorias anteriores, enquanto entre os acidentários o destaque foi para lesões de ombro, dorsopatias e sinovite e tenossinovite.

Ao analisar esse conjunto de categorias diagnósticas segundo o sexo e a idade (Tabela 6 ), encontra-se que a diferença entre as faixas etárias no geral é maior no sexo feminino ( $58 \%$ comparada a $35 \%$ entre os homens), porém a tendência dessa diferença entre as faixas etárias foi semelhante em ambos os sexos. Foi possível identificar dois grupos de categorias: aquelas nas quais a influência da idade é clara e aquelas nas quais o avanço da idade parece não representar um fator de risco para o aumento da incidência, ou ainda nas quais ser mais jovem é que representa um fator de risco. Entre as categorias mais influenciadas pela idade destacam-se as dorsopatias, lesões do ombro, mononeuropatias dos membros superiores e varizes dos membros inferiores. Em todas essas categorias a incidência entre os trabalhadores com 40 ou mais anos foi no mínimo $100 \%$ maior do que entre aqueles com menos de 40 anos. Por outro lado, entre aquelas 
Prevalência * de benefícios auxílio-doença segundo atividade econômica Cadastro Nacional de Atividades Econômicas (CNAE-classe), espécie de beneficio, sexo e idade entre trabalhadores empregados no sub-ramo carne e pescado. Brasil, 2008.

\begin{tabular}{|c|c|c|c|c|c|c|c|c|}
\hline CNAE & $\mathrm{n}$ & $<20$ ** & $20-29$ & $30-39$ & $40-49$ & $50-59$ & $\geq 60$ & Total \\
\hline \multicolumn{9}{|l|}{ B31 } \\
\hline 1011 & 8.987 & 298,4 & 757,6 & 860,7 & 1025,8 & 1031 & $1.069,9$ & 828,2 \\
\hline 1012 & 10.526 & 144,0 & 458,3 & 563,5 & 649,7 & 683,0 & 483,9 & 504,6 \\
\hline 1013 & 1.482 & 128,3 & 265,2 & 286,7 & 371,7 & 439,6 & 427,0 & 295,4 \\
\hline 1020 & 734 & 114,9 & 415,8 & 571,3 & 854,4 & 945,2 & 625,8 & 592,4 \\
\hline Total & 21.729 & 177,9 & 517,5 & 611,3 & 728,4 & 780,8 & 737,4 & 537 \\
\hline \multicolumn{9}{|l|}{ B91 } \\
\hline 1011 & 4.762 & 231,2 & 393,6 & 466,8 & 538,8 & 550,9 & 282,1 & 438,8 \\
\hline 1012 & 4.431 & 69,4 & 177,5 & 238,7 & 313,5 & 292,7 & 181,5 & 212,4 \\
\hline 1013 & 661 & 92,2 & 109,4 & 136,3 & 181,8 & 157,5 & 81,3 & 131,8 \\
\hline 1020 & 330 & 91,9 & 169,1 & 280,3 & 374,1 & 418,1 & 312,9 & 266,3 \\
\hline Total & 10.184 & 110,5 & 229,6 & 291,3 & 363,1 & 368,9 & 214,9 & 251,7 \\
\hline \multicolumn{9}{|c|}{ Masculino } \\
\hline 1011 & 8.618 & 567,0 & $1.054,3$ & $1.062,5$ & $1.231,1$ & $1.383,9$ & $1.361,3$ & $1.085,3$ \\
\hline 1012 & 6.038 & 244,3 & 477,9 & 491,1 & 617,1 & 747,8 & 650,8 & 498,7 \\
\hline 1013 & 1.305 & 246,6 & 360,5 & 359,8 & 495,8 & 495,5 & 561,3 & 389,2 \\
\hline 1020 & 483 & 255,1 & 488,7 & 702,2 & 825,5 & $1.023,0$ & 1010,4 & 663,8 \\
\hline Total & 16.444 & 335,6 & 648,8 & 668,1 & 812,5 & 964,6 & 978,2 & 681,5 \\
\hline \multicolumn{9}{|l|}{ Feminino } \\
\hline 1011 & 5.131 & 404,5 & $1.416,5$ & $1.962,0$ & $2.453,9$ & $2.448,1$ & $1.251,2$ & $1.762,8$ \\
\hline 1012 & 8.919 & 169,5 & 858,6 & $1.183,5$ & $1.456,5$ & $1.538,3$ & 754,0 & $1.019,1$ \\
\hline 1013 & 838 & 159,9 & 403,2 & 537,7 & 665,1 & 898,6 & 155,6 & 503,8 \\
\hline 1020 & 581 & 124,4 & 739,8 & $1.046,9$ & $1.727,6$ & $1.845,8$ & 792,6 & $1.136,2$ \\
\hline Total & 15.469 & 203,2 & 919,8 & $1.267,0$ & $1.583,2$ & $1.695,8$ & 779,0 & $1.117,9$ \\
\hline Total & 31.913 & 288,4 & 747 & 902,6 & $1.091,6$ & $1.149,7$ & 952,3 & 788,7 \\
\hline
\end{tabular}

* Por 10 mil trabalhadores;

** Em anos.

B31: benefício auxílio-doença previdenciário; B91: benefício auxílio-doença acidentário. 1011: abate de bovinos, equinos, ovinos e caprinos - frigoríficos; 1012: abate de suínos, aves e pequenos animais - frigoríficos; 1013: fabricação de produtos de carne e preparo de subprodutos do abate; 1020: pescado - preservação e fabricação de produtos de pescado.

categorias que apresentaram pouca diferença ou que a incidência foi maior na faixa etária $<40$ anos, merecem destaque os episódios depressivos, independentemente do sexo; fratura no nível do punho e da mão, tendinite e tenossinovite, e fratura da perna, incluindo o tornozelo (Tabela 6). Ao se fazer essa análise de forma mais específica em relação ao sexo e à idade, observou-se que entre homens jovens ( $<40$ anos) as maiores incidências decorreram das fraturas do punho e da mão (54,8\%), das dorsopatias (33\%) e das fraturas da perna, incluindo tornozelo $(31,7 \%)$, enquanto entre os homens adultos ( $\geq 40$ anos) predominaram as dorsopatias, hérnias inguinais e as lesões do ombro. Entre as mulheres as maiores incidências foram decorrentes de episódios depressivos, hemorragia do início da gravidez, e lesões do ombro, enquanto entre aquelas com 40 anos ou mais, foram as dorsopatias, lesões do ombro e as mononeuropatias dos membros superiores. Observa-se, portanto, a forte presença das lesões do ombro entre as causas clínicas de incapacidade para o trabalho, independentenebte do sexo e da idade (Tabela 6).

Ao analisar a duração dos benefícios, utilizando-se da curva de sobrevivência de Kaplan-Meier, verificou-se que os benefícios concedidos ao sexo masculino apresentaram 
Prevalência * de benefícios auxílio-doença concedidos aos trabalhadores empregados do sub-ramo de atividades de carne e pescado, segundo a atividade econômica Cadastro Nacional de Atividades Econômicas (CNAE-classe), sexo espécie de benefício. Brasil, 2008.

\begin{tabular}{lccccccccc}
\hline CNAE & \multicolumn{3}{c}{ Masculino } & \multicolumn{3}{c}{ Feminino } & & & Benefício \\
& B31 & B91 & Total & B31 & B91 & Total & B31 & B91 & Total \\
\hline 1011 & 690,0 & 395,3 & $1.085,3$ & $1.205,2$ & 557,6 & $1.762,8$ & 799,5 & 423,7 & $1.223,2$ \\
1012 & 350,3 & 148,4 & 498,7 & 718,2 & 301,0 & $1.019,1$ & 463,6 & 195,1 & 658,7 \\
1013 & 260,1 & 129,1 & 389,2 & 366,7 & 137,1 & 503,8 & 284,8 & 127,0 & 411,8 \\
1020 & 467,3 & 196,5 & 663,8 & 770,5 & 365,7 & $1.136,2$ & 558,9 & 251,3 & 810,2 \\
Total & 453,1 & 228,4 & 681,5 & 780,3 & 337,6 & $1.117,9$ & 537,0 & 251,7 & 788,7 \\
$n$ & 10.932 & 5.512 & 16.444 & 10.797 & 4.672 & 15.469 & 21.729 & 10.184 & 31.913 \\
\hline
\end{tabular}

* Por 10 mil trabalhadores.

B31: benefício auxílio-doença previdenciário; B91: beneficio auxílio-doença acidentário; 1011: abate de bovinos, equinos, ovinos e caprinos - frigoríficos; 1012: abate de suínos, aves e pequenos animais - frigoríficos; 1013: fabricação de produtos de carne e preparo de subprodutos do abate; 1020: pescado - preservação e fabricação de produtos de pescado.

maior sobrevida quando comparados ao feminino, $(\mathrm{p}<0,0001)$. Homens e mulheres apresentaram maior duração de benefício em diferentes espécies, sendo esta, entre os homens, maior entre os benefícios previdenciários, enquanto entre as mulheres foram mais longos nos acidentários. A diferença de duração entre as espécies de benefícios foram estatisticamente significativas ( $p$ $<0,0001)$ em ambos os sexos.

O ramo pescados apresentou maior sobrevida de benefícios auxílio-doença, independentemente do sexo do trabalhador e da espécie de benefício $(p<0,0001)$.

Mais de três quartos (80\%) dos benefícios auxílio-doença receberam alta em até 100 dias. No geral, a mediana da duração dos benefícios foi de 52 dias, sendo esta maior entre os benefícios auxílio-doença acidentários (55 dias), e no ramo pescado, porém sem significância estatística.

\section{Discussão}

Este estudo mostrou que o coeficiente de incidência de benefícios auxílio-doença no ramo de atividade econômica de carne e pescado em 2008 foi quase o dobro daquela apresentada pela média dos trabalhadores empregados no Brasil 3 , contudo são mais próximas das taxas de incidência de benefícios encontradas em países europeus como a Holanda $11[1050,0]$ e na França 12 [1370,0], especialmente quando comparadas com as incidências apresentadas por atividade econômica como o abate de bovinos, equinos, ovinos e caprinos - e preservação e fabricação de produtos de pescado - Pescado.

Quando considerados os benefícios auxíliodoença de origem ocupacional (acidentária) no ramo Carne e Pescado, de certa forma, corrobora as particularidades e variedade dos fatores de risco presentes nesse ramo de atividade ao apresentar incidência de benefícios acidentários superior 2,7 vezes à média nacional dos trabalhadores empregados 7,9.

As maiores incidências de benefícios nas UFs da Região Sul podem decorrer tanto de uma maior notificação quanto de condições inadequadas de trabalho. Um importante fator é a existência de uma maior concentração de grandes empresas (incluindo certo monopólio) do ramo nessa região geográfica. Algumas empresas da Região Sul do ramo de carnes e pescado têm, inclusive, sido alvo de denúncias por parte das instituições fiscalizadoras e sindicais em relação às péssimas condições/relações de trabalho, apontando uma verdadeira epidemia de agravos à saúde dos trabalhadores. Outras possibilidades que ajudam a explicar esse quadro é a maior conscientização dos trabalhadores, melhor nível de escolaridade, sindicalização e acesso à previdência social. Em outras regiões e unidades federativas onde ocorreram menores incidências de benefícios é possível que elas sofram o efeito dos vários filtros socioeconômicos 7,12. Quadro semelhante foi encontrado em estudo brasileiro de base populacional que incluiu todos os trabalhadores empregados no Brasil, o qual avaliou os benefícios auxílio-doença decorrente de doenças 
Tabela 4

Prevalência * de benefícios auxílios-doença segundo os capítulos da 10a revisão da Classificação Internacional de Doenças (CID-10), sexo e espécie de benefício no sub-ramo de carne e pescado. Brasil, 2008.

\begin{tabular}{|c|c|c|c|c|c|c|c|c|c|}
\hline \multirow[t]{2}{*}{ Capítulo (CID-10) } & \multicolumn{3}{|c|}{ Masculino } & \multicolumn{3}{|c|}{ Feminino } & \multicolumn{3}{|c|}{ Benefício } \\
\hline & B31 & B91 & Total & B31 & B91 & Total & B31 & B91 & Total \\
\hline XIX & 176,9 & 133,5 & 310,5 & 82,1 & 62,9 & 145 & 133,6 & 101,1 & 234,7 \\
\hline XIII & 76,7 & 72,7 & 149,4 & 133,8 & 191,9 & 325,7 & 91,5 & 109,0 & 200,5 \\
\hline V & 47,7 & 4,0 & 51,7 & 160,4 & 25,5 & 185,9 & 83,3 & 11,1 & 94,4 \\
\hline$X V$ & 0,0 & 0,0 & 0,0 & 92,1 & 0,1 & 92,1 & 92,1 & 0,0 & 92,1 \\
\hline$X I$ & 54,3 & 5,6 & 59,8 & 59,3 & 2,7 & 62,0 & 52,6 & 4,3 & 56,9 \\
\hline IX & 27,8 & 2,6 & 30,3 & 58,2 & 6,8 & 65,0 & 36,5 & 3,9 & 40,3 \\
\hline VI & 7,0 & 5,1 & 12,1 & 18,2 & 42,5 & 60,7 & 10,4 & 17,6 & 28,0 \\
\hline XIV & 9,5 & 0,1 & 9,6 & 63,5 & 0,0 & 63,5 & 27,4 & 0,1 & 27,4 \\
\hline II & 7,4 & 0,1 & 7,5 & 46,9 & 0,1 & 47,0 & 20,4 & 0,1 & 20,5 \\
\hline XII & 9,1 & 1,3 & 10,4 & 13,6 & 1,4 & 15,0 & 10,1 & 1,2 & 11,3 \\
\hline VII & 9,4 & 1,3 & 10,8 & 7,9 & 0,5 & 8,5 & 8,4 & 1,0 & 9,3 \\
\hline I & 9,0 & 1,1 & 10,1 & 7,2 & 0,6 & 7,8 & 7,9 & 0,8 & 8,7 \\
\hline$X$ & 6,3 & 0,4 & 6,7 & 9,8 & 1,0 & 10,8 & 7,1 & 0,6 & 7,7 \\
\hline$X X I$ & 3,2 & 0,4 & 3,6 & 10,6 & 1,2 & 11,8 & 5,6 & 0,6 & 6,2 \\
\hline IV & 1,6 & 0,0 & 1,6 & 3,5 & 0,0 & 3,5 & 2,2 & 0,0 & 2,2 \\
\hline VIII & 1,6 & 0,1 & 1,7 & 2,8 & 0,0 & 2,8 & 1,9 & 0,1 & 2,0 \\
\hline III & 0,9 & 0,1 & 1,0 & 1,7 & 0,0 & 1,7 & 1,1 & 0,0 & 1,2 \\
\hline XVII & 0,8 & 0,0 & 0,8 & 0,5 & 0,0 & 0,5 & 0,6 & 0,0 & 0,6 \\
\hline$X X$ & 0,0 & 0,0 & 0,0 & 0,0 & 0,1 & 0,1 & 0,0 & 0,0 & 0,0 \\
\hline Outros & 3,8 & 0,0 & 3,9 & 8,2 & 0,4 & 8,6 & 5,1 & 0,2 & 5,2 \\
\hline Total & 453,1 & 228,4 & 681,5 & 780,3 & 337,6 & $1.117,9$ & 537 & 251,7 & 788,7 \\
\hline \pm & 10,9 & 7,8 & 13,2 & 18,6 & 12,5 & 21,9 & 9,1 & 6,4 & 10,9 \\
\hline
\end{tabular}

* Por 10 mil trabalhadores.

B31: beneficio previdenciário; B91: benefício acidentário

Capítulos CID-10: I - Algumas doenças infecciosas e parasitárias; II - Neoplasias (tumores); III - Doenças do sangue e dos órgãos hematopoiéticos e alguns transtornos imunitários; IV - Doenças endócrinas, nutricionais e metabólicas; $V$ - Transtornos mentais e comportamentais; VI - Doenças do sistema nervoso; VII - Doenças do olho e anexos; VIII - Doenças do ouvido e da apófise mastoide; IX - Doenças do aparelho circulatório; X - Doenças do aparelho respiratório; XI - Doenças do aparelho digestivo; XII - Doenças da pele e do tecido subcutâneo; XIII - Doenças do sistema osteomuscular e do tecido conjuntivo; XIV - Doenças do aparelho geniturinário; XV - Gravidez, parto e puerpério; XVI - Algumas afecções originadas no período perinatal; XVII - Malformações congênitas, deformidades e anomalias cromossômicas; XVIII - Sintomas, sinais e achados anormais de exames clínicos e de laboratório, não classificados em outra parte; XIX - Lesões, envenenamento e algumas outras consequências de causas externas; XX - Causas externas de morbidade e de mortalidade; XXI - Fatores que influenciam o estado de saúde e o contato com os serviços de saúde.

osteomusculares ${ }^{8}$. Essa semelhança corrobora a tese de que esse é um fenômeno relacionado tanto à concessão de benefícios quanto a quadros de doenças ou ramos de atividades específicos.

As altas taxas de desemprego em 2008 e a falta de sindicatos fortes para proteger os direitos do trabalhador poderiam ter levado alguns trabalhadores a continuar trabalhando ou retornar ao trabalho antes de 15 dias, enquanto ainda doentes, a fim de evitar o benefício. A alta probabilidade de demissão no retorno ao trabalho, especialmente nos casos de benefícios previdenciários, e a dificuldade em encontrar outro emprego, em particular durante períodos de recessão econômica, também são importantes na decisão dos trabalhadores em solicitar ou não um benefício junto à Previdência Social, caracterizando assim o presenteísmo.

Apesar de as taxas desses sub-ramos de atividade se apresentarem bem acima da média nacional, é possível que ainda estejam subnotificadas. Tal suspeita se fortalece ao comparar essas taxas com as apresentadas em países desenvolvidos 11,12 . Se por um lado a instituição do 
Tabela 5

Prevalência * de benéficos auxílios-doença segundo a categoria diagnóstica CID-10, a espécie de benefício e a atividade econômica Cadastro Nacional de Atividades Econômicas (CNAE-classe) no sub-ramo de carne e pescado. Brasil, 2008.

\begin{tabular}{|c|c|c|c|c|c|c|c|c|c|c|c|}
\hline \multirow{2}{*}{$\begin{array}{l}\text { Diagnóstico } \\
\text { (CID-10) }\end{array}$} & \multicolumn{5}{|c|}{ Benefício previdenciário (B31) } & \multicolumn{5}{|c|}{ Benefício acidentário (B91) } & \multirow{2}{*}{$\begin{array}{l}\text { Total } \\
\text { Total }\end{array}$} \\
\hline & 1011 & 1012 & 1013 & 1020 & Total & 1011 & 1012 & 1013 & 1020 & Total & \\
\hline O20 & 87,3 & 52,6 & 25,8 & 15,6 & 55,3 & 0,0 & 0,0 & 0,0 & 0,0 & 0,0 & 55,3 \\
\hline M54 & 39,2 & 18,6 & 15,8 & 44,9 & 24,8 & 45,8 & 18,6 & 11,7 & 41,1 & 26,0 & 50,8 \\
\hline F32 & 37,3 & 44,2 & 16,7 & 18,3 & 37,9 & 6,1 & 8,9 & 1,3 & 0,0 & 6,9 & 44,8 \\
\hline M75 & 12,3 & 8,7 & 6,3 & 10,7 & 9,5 & 42,3 & 27,9 & 11,0 & 30,5 & 29,8 & 39,2 \\
\hline S62 & 31,3 & 13,9 & 11,1 & 15,2 & 18,4 & 32,7 & 11,6 & 10,8 & 17,5 & 17,6 & 36,0 \\
\hline M65 & 11,7 & 6,8 & 6,5 & 14,5 & 8,4 & 38,2 & 20,0 & 10,6 & 45,7 & 24,7 & 33,0 \\
\hline S82 & 28,0 & 12,2 & 9,0 & 17,5 & 16,4 & 11,4 & 4,1 & 4,8 & 4,6 & 6,2 & 22,6 \\
\hline G56 & 5,5 & 4,7 & 2,7 & 0,0 & 4,5 & 18,8 & 19,3 & 4,8 & 9,9 & 17,0 & 21,5 \\
\hline 183 & 19,5 & 17,0 & 7,7 & 12,2 & 16,3 & 2,7 & 2,2 & 1,0 & 1,5 & 2,2 & 18,5 \\
\hline K40 & 29,3 & 10,8 & 7,5 & 17,5 & 15,7 & 5,2 & 1,6 & 1,0 & 1,5 & 2,5 & 18,3 \\
\hline S52 & 19,1 & 7,2 & 5,8 & 8,4 & 10,4 & 11,5 & 4,2 & 2,9 & 8,4 & 6,2 & 16,6 \\
\hline S92 & 15,9 & 6,8 & 5,4 & 9,1 & 9,2 & 11,0 & 3,9 & 5,8 & 3,0 & 6,1 & 15,3 \\
\hline S83 & 19,2 & 9,0 & 4,4 & 3,8 & 11,1 & 8,2 & 2,6 & 1,0 & 3,0 & 4,0 & 15,0 \\
\hline M51 & 15,7 & 7,9 & 6,9 & 12,9 & 10,1 & 6,9 & 2,4 & 3,3 & 3,8 & 3,8 & 13,9 \\
\hline S61 & 5,7 & 2,2 & 1,9 & 1,5 & 3,1 & 22,3 & 6,7 & 5,2 & 4,6 & 10,8 & 13,9 \\
\hline S42 & 18,4 & 7,4 & 5,8 & 5,3 & 10,2 & 6,4 & 2,4 & 2,3 & 1,5 & 3,5 & 13,7 \\
\hline F33 & 8,4 & 14,3 & 2,3 & 5,3 & 10,8 & 1,1 & 2,1 & 0,6 & 0,0 & 1,5 & 12,3 \\
\hline 060 & 19,9 & 11,3 & 5,4 & 0,0 & 12,0 & 0,0 & 0,0 & 0,0 & 0,0 & 0,0 & 12,0 \\
\hline M77 & 3,4 & 2,8 & 1,5 & 0,0 & 2,7 & 10,5 & 6,9 & 3,5 & 4,6 & 7,4 & 10,1 \\
\hline K35 & 16,2 & 6,8 & 5,4 & 8,4 & 9,3 & 0,7 & 0,5 & 0,0 & 0,8 & 0,5 & 9,8 \\
\hline Outros & 435,6 & 237,6 & 152,0 & 347,2 & 285,2 & 141,8 & 49,1 & 45,7 & 69,3 & 75,1 & 360,2 \\
\hline Total & 799,5 & 463,6 & 284,8 & 558,9 & 537,0 & 423,7 & 195,1 & 127,0 & 251,3 & 251,7 & 788,7 \\
\hline
\end{tabular}

* Por 10 mil trabalhadores.

CNAE: 1011 - abate de bovinos, equinos, ovinos e caprinos - frigoríficos; 1012 - abate de suínos, aves e

pequenos animais - frigoríficos; 1013 - fabricação de produtos de carne e preparo de subprodutos do abate;

1020 - pescado - preservação e fabricação de produtos de pescado. F32: episódios depressivos; F33: transtorno depressivo recorrente; G56: mononeuropatias dos membros superiores; 183: varizes dos membros inferiores; K35: doenças do apêndice; K40: hérnia inguinal; M51: outros transtornos de discos intervertebrais; M54: dorsalgia; M65: sinovite e tenossinovite; M75: lesões do ombro; M77: outras entesopatias; O20: hemorragia do início da gravidez; O60: trabalho de parto pré-termo; S42: fratura do ombro e do braço; S52: fratura do antebraço; S61: ferimento do punho e da mão; S62: fratura no nível do punho e da mão; S82: fratura da perna, incluindo tornozelo; S83: luxação, entorse e distensão das articulações e dos ligamentos do joelho; S92: fratura do pé (exceto do tornozelo).

Nexo Técnico Epidemiológico Previdenciário (NTEP) 7,13 contribuiu para o aumento da caracterização da relação trabalho-doença, aumentando os benefícios acidentários, por outro, incentivou os empregadores a instituírem medidas "de saúde e segurança no trabalho" caracterizadas pela premiação coletiva de grupos específicos para os chamados "acidente zero", "zero dias perdidos", “afastamento zero” etc., nos quais está embutido o conceito da responsabilidade coletiva pelo afastamento.

A grande diferença (48\%) entre as incidências de benefício auxílio-doença encontradas nas CNAE Carne-1011 e Pescado-1020 pode decor- rer das características dos processos produtivos, da organização de trabalho, das características socioeconômicas e educacionais dos trabalhadores, dos sistemas de compensação e gratificações e das relações de poder entre empregador/ empregado, caracterizadas particularmente pela presença/ausência de sindicatos fortes, oferta de empregos e grau de especificidade da atividade.

A incapacidade para o trabalho tem sido associada a diversos fatores psicossociais como a elevada demanda de trabalho físico, tensão psicológica, violência e angústia, recursos inadequados para o desenvolvimento do trabalho e a percepção de baixa qualidade de saúde 
Incidência * dos benefícios auxílio-doença segundo a categoria diagnóstica da 10ạ revisão da Classificação Internacional de Doenças (CID-10), sexo, idade e espécie de benefício no sub-ramo de carne e pescado. Brasil, 2008.

\begin{tabular}{|c|c|c|c|c|c|c|c|}
\hline \multirow[t]{2}{*}{ CID-10 } & \multicolumn{3}{|c|}{ Masculino } & \multicolumn{3}{|c|}{ Feminino } & \multirow[t]{2}{*}{ Total } \\
\hline & $<40$ & $\geq \mathbf{4 0}$ & Total & $<40$ & $\geq \mathbf{4 0}$ & Total & \\
\hline O20 & - & - & - & 66,9 & 5,7 & 55,3 & 55,3 \\
\hline M54 & 33,0 & 87,5 & 44,9 & 54,1 & 139,5 & 70,3 & 50,8 \\
\hline F32 & 15,2 & 14,2 & 15,0 & 109,2 & 85,7 & 104,8 & 44,8 \\
\hline M75 & 17,4 & 40,5 & 22,5 & 65,5 & 118,9 & 75,6 & 39,2 \\
\hline S62 & 54,8 & 39,4 & 51,4 & 14,6 & 19,8 & 15,6 & 36,0 \\
\hline M65 & 18,1 & 17,6 & 18,0 & 60,3 & 86,1 & 65,2 & 33,0 \\
\hline S82 & 31,7 & 27,8 & 30,9 & 11,4 & 15,6 & 12,2 & 22,6 \\
\hline G56 & 5,3 & 10,0 & 6,3 & 41,6 & 96,0 & 51,9 & 21,5 \\
\hline 183 & 6,4 & 16,5 & 8,6 & 30,7 & 75,1 & 39,1 & 18,5 \\
\hline K40 & 21,7 & 53,2 & 28,6 & 2,9 & 6,5 & 3,5 & 18,3 \\
\hline S52 & 23,2 & 16,3 & 21,7 & 10,3 & 12,6 & 10,7 & 16,6 \\
\hline S92 & 20,1 & 17,8 & 19,6 & 9,5 & 15,6 & 10,7 & 15,3 \\
\hline S83 & 20,5 & 16,7 & 19,6 & 9,4 & 11,1 & 9,7 & 15,0 \\
\hline M51 & 10,9 & 27,5 & 14,5 & 9,6 & 40,4 & 15,5 & 13,9 \\
\hline S61 & 17,7 & 18,2 & 17,8 & 9,2 & 11,4 & 9,6 & 13,9 \\
\hline S42 & 20,8 & 14,6 & 19,4 & 6,3 & 5,3 & 6,1 & 13,7 \\
\hline F33 & 3,7 & 4,9 & 3,9 & 29,3 & 28,6 & 29,2 & 12,3 \\
\hline O60 & - & - & - & 14,6 & 0,8 & 12,0 & 12,0 \\
\hline M77 & 4,5 & 9,3 & 5,5 & 14,6 & 42,7 & 19,9 & 10,1 \\
\hline K35 & 12,4 & 6,4 & 11,1 & 10,5 & 4,2 & 9,3 & 9,8 \\
\hline Outros & 295,6 & 417,3 & 322,2 & 428,2 & 682,9 & 476,5 & 355,1 \\
\hline Total & 632,8 & 855,5 & 681,5 & $1.007,0$ & $1.592,2$ & $1.117,9$ & 788,7 \\
\hline
\end{tabular}

B31: benefício auxílio-doença previdenciário; B91: benefício auxílio-doença acidentário; O20: hemorragia do início da gravidez; M54: dorsalgia; F32: episódios depressivos; M75: lesões do ombro; S62: fratura ao nível do punho e da mão; M65: sinovite e tenossinovite; S82: fratura da perna, incluindo tornozelo; G56: mononeuropatias dos membros superiores; 183: varizes dos membros inferiores; K40: hérnia inguinal; S52: fratura do antebraço; S92: fratura do pé (exceto do tornozelo); S83: luxação, entorse e distensão das articulações e dos ligamentos do joelho; M51: outros transtornos de discos intervertebrais; S61: ferimento do punho e da mão; S42: fratura do ombro e do braço; F33: transtorno depressivo recorrente; O60: trabalho de parto pré-termo; M77: outras entesopatias; K35-K38: doenças do apêndice.

física e psicológica, longa jornada de trabalho e trabalho em turnos com comprometimento do ciclo circadiano e baixo nível educacional. Esses fatores de risco associados ao estresse e outros transtornos mentais como depressão e ansiedade contribuem sobremaneira para a deterioração da funcionalidade física e mental 5,6.

Em relação às elevadas incidências de lesões e de doenças osteomusculares identificadas nesses ramos de atividades, é possível que fatores como a alta velocidade das linhas de produção de carne e frango e seus subprodutos, o manuseio de ferramentas afiadas, o esforço físico elevado, combinado com movimentos repetitivos e longas jornadas contribuam diretamente para a ocorrência delas 14,15.
A automação e a mecanização em frigoríficos são restritas, em sua maioria, destinadas apenas ao levantamento e movimentação de animais abatidos, mas o corte e procedimentos de separação das partes permanecem como um trabalho físico braçal, com grande sobrecarrega muscular, sobretudo dos membros superiores e da região escapular do trabalhador 16,17.

Todo o manuseio de produtos perecíveis, em especial em carne e pescado, obriga os trabalhadores a permanente corrida contra o relógio, visando diminuir o risco de deterioração e contaminação do produto, o que, associado a fatores como ambientes com inadequados níveis de iluminação, ruído e temperatura, frequente manipulação de instrumentos cortantes (faca, mo- 
tosserra) e a exigência de intensa concentração durante a atividade pode influenciar, em grande escala, o quadro de saúde e segurança dos trabalhadores, e consequentemente, as taxas de incapacidade para o trabalho 17 . A organização do trabalho e o processo produtivo nos ramos de atividade estudados não permitem que o trabalhador tenha controle sobre seu modo de trabalhar, podendo tornar o trabalho extremamente estressante, o que contribui para o aumento do risco de agravos físicos e mentais no trabalhador, especialmente para a ocorrência de lesões e doenças do sistema osteomuscular e do tecido conjuntivo identificadas neste estudo $3,4,8,18$.

A elevada representatividade das Lesões, doenças do sistema osteomuscular e do tecido conjuntivo e transtornos mentais e comportamentais, assim como a maior prevalência de lesões entre os homens e de doenças osteomusculares e de mentais entre as mulheres, encontradas neste estudo, são corroborados tanto pela literatura nacional 3,7,8,9 quanto internacional 4,19,20,21,22,23.

Tanto os transtornos mentais quanto as doenças osteomusculares apresentam em sua maioria etiologia multifatorial, envolvendo características de natureza genética e psicossocial, além de fatores biomecânicos e podem agir como agentes sinérgicos no desenvolvimento/agravamento dessas enfermidades.

No ramo de atividade carne e pescado, dentre os fatores biomecânicos sabidamente presentes no processo de trabalho e associados ao desenvolvimento de distúrbios osteomusculares, destacam-se a alta repetitividade, elevação das mãos associada a peso e força, ausência ou limitação de pausas, alta demanda psicológica, baixo apoio social, movimentos de flexão/extensão, e de pronosupinação, assim como manuseio de ferramentas vibratórias 24 .

O estresse relacionado com o trabalho pode contribuir para o aumento da criminalidade e ocorrências de outros transtornos mentais como depressão, ansiedade e distúrbios de humor, alcoolismo, abuso de drogas/dependência 25.

A natureza multifatorial e multidimensional da dor musculoesquelética tem sido objeto de vários estudos que demonstram que o estresse psicológico e outros sintomas somáticos estão relacionados com queixas de dores inespecíficas. Andersen et al. 26 estudaram os fatores de risco no local de trabalho para dores no ombro e pescoço em trabalhadores dinamarqueses. Destacaram-se os fatores físicos: as posturas inadequadas (o trabalho sentado e a flexão do pescoço), as tarefas manuais repetitivas (esforços repetitivos com o ombro elevado), a carga física elevada, e os fatores psicossociais: alto nível de estresse mental (demanda excessiva de trabalho, baixo controle sobre o trabalho, altos níveis de angústia). Esses fatores de risco são preditores do aparecimento de dores no pescoço e nos ombros nos trabalhadores.

Ao considerar apenas os benefícios acidentários, este estudo apresentou taxas mais elevadas entre as mulheres em carne e pescado, discordando dos resultados de outras pesquisas 2,7,9,27. Segundo Nelson et al. 27, a carga de doenças, por causa da exposição ocupacional, nos países em desenvolvimento é maior para os homens, refletindo diferenças na exposição e atividades econômicas. A elevada incidência de benefícios auxílio-doença acidentários entre as trabalhadoras na atividade econômica de carne e pescado pode estar associada ao tipo de trabalho manual, principalmente aqueles com movimentos repetitivos e rotação do ombro e do tronco 1,2,8,9,16,17,26.

A maior ocorrência de incapacidade para o trabalho entre as mulheres e o aumento de tal ocorrência com a idade, encontrada neste estudo, é confirmada tanto pela literatura nacional 3,7,8,9 quanto internacional 4,11,19,20,21,22,23.

Em geral, nessas atividades, homens e mulheres estão envolvidos em trabalhos com características de exigências e demandas mentais e emocionais distintas, que podem contribuir para o aumento do risco de incapacidade para o trabalho e, assim, para as diferenças de sexo observadas no afastamento do trabalho 22.

Existem diferenças importantes nas condições de trabalho, sejam elas envolvendo aspectos físicos, psicológicos, familiares, ergonômicos, econômicos e organizacionais que podem explicar o diferencial entre os sexos 7,11,12,22. Outros fatores específicos descritos por pesquisadores franceses 11 e holandeses 12 como exposição a abuso e violências doméstica, sexual, sejam físicas e/ ou psicológicas, baixa renda, responsabilidade exclusiva pela criação e manutenção dos filhos, podem contribuir para essa maior incidências de benefícios entre as mulheres.

A maior incidência de transtornos de discos intervertebrais e outras entesopatias, assim como de lesões musculoesqueléticas entre as mulheres encontradas no presente estudo foram também identificadas em outros estudos 7,8,9,12.

A maior incidência de sinovite e tenossinovite apresentada pelas trabalhadoras do ramo pescado pode estar associada ao trabalho repetitivo e em baixas temperaturas comumente presentes nessa atividade. Por outro lado, a elevada prevalência de episódios depressivos entre os homens nesse ramo de atividade pode ser influenciada pelas características das atividades entre os sexos. Enquanto entre as mulheres predominam atividades de limpeza e processamento do pescado, entre os homens inclui, entre outras, a 
atividade de pesca, o que pode ser caracterizado por longos e repetidos períodos de isolamento social, além do risco constante de intempéries climáticas e outros perigos presentes nos rios e oceanos. O isolamento social tem sido apontado como importante fator de risco para o desenvolvimento/agravamento de episódios depressivos 28.

A elevada incidência de neoplasias entre o sexo feminino e a ausência de nexo técnico nesse grupo de doenças pode ser melhor entendido ao considerar uma série de aspectos já destacados por outros autores. Corrêa 29 infere a existência de um verdadeiro silêncio epidemiológico no câncer relacionado ao trabalho no Brasil. A invisibilidade do câncer relacionado ao trabalho pode ser avaliada pela irrisória participação das neoplasias relacionadas ao trabalho dentre os auxílios-doença acidentários concedidos pela Previdência Social. O lapso de tempo e o desconhecimento do assunto dificultam o nexo técnico e o seu registro adequado no sistema de notificação ${ }^{30}$. Acrescenta-se a esses fatores o fato de a incidência de câncer na população geral ser maior no sexo feminino do que no masculino, podendo influenciar muitos profissionais da saúde a perceberem o sexo (feminino) como um fator de risco mais importante do que a potencial exposição a agentes químicos ou outros riscos ocupacionais. Dessa forma, contribuem para aumentar a subnotificação da relação da doença com o trabalho.

Os homens adultos ( $\geq 40$ anos) apresentaram maiores incidências em relação aos homens adultos jovens ( $<40$ anos). As incidências atingiram um pico de idade na faixa de 50-59 anos, seguindo os achados de trabalho holandês 11. O aumento das prevalências dos benefícios e o envelhecimento que foi encontrada neste estudo reiteram pesquisas anteriores 7,12,16,17, demonstrando que a idade é forte fator de risco para a incapacidade para o trabalho.

O efeito da idade sobre a incapacidade para o trabalho parece ser mais claro para algumas categorias diagnósticas, tais como distúrbios osteomusculares, neoplasias e doenças do aparelho circulatório, em que o aumento com idade é evidente. Todavia, os transtornos mentais e lesões apresentaram padrões de idade de distribuição distintos 7 .

Segundo estudo brasileiro 8 , a prevalência de benefícios decorrentes de distúrbios osteomusculares foi de 93,6, sendo os quatro benefícios mais comuns aqueles devidos à lombalgia, discopatia intervertebral, sinovite/tenossinovite e distúrbios do ombro. A prevalência aumentou com a idade. A menor idade é fator de risco para doenças osteomusculares como dor lombar enquanto a idade avançada é fator de risco para dor em ombro e de pulso/mão (incluindo sinovite/tenossinovite).

Quando se analisaram os benefícios segundo o diagnóstico CID específico, o sexo, a idade e a espécie de beneficio, chamou a atenção a predominância de benefícios B91 para vários agravos à saúde, com destaque para as lesões do ombro, dorsopatias, sinovite e tenossinovite, fratura no nível do punho e da mão e síndrome do túnel do carpo. Resultados similares foram publicados por diversos autores nacionais e internacionais $1,2,8,17,31$.

A mediana da duração dos benefícios foi de 52 dias de afastamento do trabalho. Lipton et al. 32 estimaram que a mediana da duração dos benefícios nos Estados Unidos no ano de 2010 foi de 42 dias. Um estudo brasileiro ${ }^{9}$ constatou a média de 59 dias em benefícios acidentários no Brasil no ano de 2008.

Comparativamente à pesquisa de Elders et al. 17, que estudou a duração de afastamentos em trabalhadores braçais holandeses, utilizando curvas de Kaplan Mayer, evidenciando que 76\% dos trabalhadores haviam retornado ao trabalho em 35 dias, a mediana brasileira foi superior. Porém, essa diferença entre as durações apresentadas entre Brasil e Holanda pode decorrer da diferença entre os sistemas de pagamento de absenteísmo-doença/benefícios. Enquanto no Brasil o empregador paga os primeiros 15 dias de afastamento e a partir desse período essa responsabilidade passa para a previdência social, na Holanda, o empregador paga o benefício auxílio-doença por até dois anos, e no primeiro ano corresponde a $100 \%$ dos vencimentos. Baseado nesse elevado encargo, o empregador acompanha o tratamento e a recuperação do trabalhador, visando otimizar o retorno ao trabalho, o que inclui perícia com o médico do trabalho a cada 4-6 semanas ${ }^{11}$. Vale ressaltar que na Holanda os auxílios-doença são computados apenas a partir do 280 dia de afastamento ${ }^{11}$. Se forem considerados os períodos de benefício nos dois países, observa-se que a diferença entre as durações totais do afastamento é pequena (63 dias na Holanda $\mathrm{x}$ 67 dias no Brasil).

Os homens apresentaram mais benefícios auxílio-doença de longa duração, enquanto entre as mulheres predominaram os afastamentos de curta duração. Tais achados de benefícios auxílio-doença corroboraram com o estudo dos benefícios concedidos pelo INSS no Brasil em 2008 7. Resultados semelhantes foram também encontrados em estudos europeus 22,33.

Entre os homens, a duração foi maior entre os benefícios previdenciários - B31 (55 dias) do que entre os acidentários - B91, ocorrendo o contrário entre as mulheres, dentre as quais os 
benefícios acidentários (60 dias) apresentaram maior duração.

Ao analisar a duração dos benefícios, comparando entre si os CNAE-classe, foi encontrada a constante predominância da CNAE 1020 sobre os outros. A preservação do pescado e fabricação de produtos de pescado [CNAE 1020] apresentou sobrevida maior, isto é, uma duração maior de benefícios (61 dias), podendo indicar uma maior gravidade das doenças nessa atividade econômica.

As empresas com altas taxas de exigência, percepção da alta demanda de trabalho, baixo controle de trabalho e apoio social deficitário no local de trabalho foram associadas com a recuperação mais tardia e lenta das doenças incapacitantes, como lesões de ombro. Mas isso pode ser uma consequência e não uma causa da doença 4 .

A alta demanda de trabalho físico e os sintomas depressivos moderados a graves são fatores associados à maior duração da incapacidade para o trabalho e benefícios concedidos. Sabe-se que a violência e o medo no trabalho constituem importantes fatores de risco para diversas doenças mentais e a violência, podendo contribuir para aumento da prevalência e da gravidade de um transtorno mental, resultando em maior duração de benefícios por incapacidade observada entre os trabalhadores em frigoríficos e matadouros 3 .

Outros fatores não estritamente médicos, como posição socioeconômica baixa, idade abaixo de 39 anos, expectativa alta de retorno ao trabalho e reposição de renda pelo INSS menor ou igual a $100 \%$ (salário mínimo), podem justificar em parte a menor duração dos benefícios com consequente retorno ao trabalho ${ }^{34}$. A associação entre a incapacidade para o trabalho e a idade tem sido reportada na maioria dos estudos e reflete tanto características intrínsecas quanto extrínsecas. Entre as intrínsecas destacam-se as comorbidades, decréscimo hormonal e alteração nas características de colágeno e elastina, típicas do envelhecimento humano 35 .

A maior limitação desta pesquisa está relacionada à população de estudo, que não compreendeu a totalidade da população trabalhadora, na medida em que excluiu os trabalhadores autônomos, os servidores públicos e os trabalhadores informais.

\section{Conclusões}

A atividade econômica carne e pescado possui importantes características ocupacionais que contribuem para as elevadas demandas de benefícios auxílio-doença junto à Previdência Social.

As taxas de incidência de benefícios apontam para fortes indícios da precarização do emprego e das condições de trabalho, e a maior duração de benefícios pode indicar tanto uma maior gravidade das doenças nesses setores produtivos, quanto uma assistência à saúde mais precária do que em outros ramos industriais.

Este trabalho fornece informações que podem ser úteis na implantação de políticas previdenciárias e de saúde do trabalhador a fim de reduzir as incapacidades para o trabalho e as consequências econômicas e sociais decorrentes. 


\section{Resumen}

El objetivo del presente estudio fue identificar factores asociados a la incidencia de concesión prestaciones sociales por enfermedad a los trabajadores del sector cárnico y pesquero en Brasil. Se estudiaron las prestaciones sociales por enfermedad, concedidas por el Seguro Social, a los empleados de los sectores anteriormente mencionados durante el año 2008. Las incidencias por cada 10 mil empleados fueron estratificadas por sexo, edad, diagnóstico, actividad económica, departamento, tipo y duración de las prestaciones por enfermedad. Se concedieron 31.913 prestaciones sociales por enfermedad, con una incidencia de 788,7. El colectivo perteneciente a mataderos de ganado vacuno, equino, bovino y caprino presentó la mayor incidencia $(1.223,2)$ y el de pesca la mayor duración (61 días de prestaciones sociales por enfermedad). Las mujeres presentaron una mayor incidencia, mientras los hombres una mayor duración de las prestaciones por enfermedad. Los grupos de lesiones, enfermedades osteomusculares y trastornos mentales representaron un $67,2 \%$ de los beneficios de auxilio por enfermedad. Los diagnósticos más incidentes fueron las dorsopatías, hemorragias en el principio de la gestación y episodios depresivos, según las ramas de la actividad ejercida, el sexo y la edad son importantes factores, sugiriendo precariedad en las condiciones de trabajo.

Seguridad Social; Salarios y Beneficios; Salud Laboral; Mataderos; Industria Pesquera

\section{Colaboradores}

H. R. Jakobi, A. Barbosa-Branco, L. F. Bueno, R. G. M Ferreira e L. M. A. Camargo contribuíram na concepção, interpretação, redação e aprovação da versão final do artigo.

\section{Referências}

1. Campoamor MM. Estudo da ocorrência de acidentes entre trabalhadores de uma indústria frigorífica do Estado de São Paulo [Dissertação de Mestrado]. Ribeirão Preto: Universidade de São Paulo; 2006.

2. Vasconcelos MC, Pignatti MG, Pignatti WA. Emprego e acidentes de trabalho na indústria frigorífica em áreas de expansão do agronegócio. Mato Grosso, Brasil. Saúde Soc 2009; 18:662-72.

3. Barbosa-Branco A, Bültmann U, Steenstra I. Sickness benefit claims due to mental disorders in Brazil: associations in a population-based study. Cad Saúde Pública 2012; 28:1854-66.
4. Bonde JP, Mikkelsen S, Andersen JH, Fallentin N, Baelum J, Svendsen SW, et al. Prognosis of shoulder tendonitis in repetitive work: a follow up study in a cohort of Danish industrial and service workers. Occup Environ Med 2003; 60:E8.

5. Dejours C, Abdoucheli E, JAyet C. Psicodinâmica do trabalho. São Paulo: Editora Atlas; 1994.

6. Khalt M, Chau N. Social disparities in musculoskeletal disorders and associated mental malaise: findings from a population-based survey in France. Scand J Public Health 2010; 38:495-501. 
7. Barbosa-Branco A, Souza WR, Steenstra IA. Incidence of work and non-work related disability claims in Brazil. Am J Ind Med 2011; 54:858-71.

8. Vieira ER, Albuquerque-Oliveira PR, BarbosaBranco A. Work disability benefits due to musculoskeletal disorders among Brazilian private sector workers. BMJ Open 2011; 1:000003.

9. Almeida PCA, Barbosa-Branco A. Acidentes de trabalho no Brasil: prevalência, duração e despesa previdenciária dos auxílios-doença. Rev Bras Saúde Ocup 2011; 36:195-207.

10. Instituto Brasileiro de Geogranfia e Estatística. Cadastro Nacional de Atividades Econômicas (CNAE 2.0). http://www.ibge.gov.br/home/estatistica/economia/classificacoes/cnae2.0_subclasses/default. shtm (acessado em 12/Abr/2010).

11. Roelen CAM, Koopmans PC, Hoedeman R, Bültmann U, Groothoff JW, van der Klink JJ. Trends in the incidence of sickness absence due to common mental disorders between 2001 and 2007 in the Netherlands. Eur J Public Health 2009; 19:625-30.

12. Ferrie JE, Vahtera J, Kivimäki M, Westerlund $H$, Melchior M, Alexanderson K et al. Diagnose-specific sickness absence and all cause mortality in the GAZEL study. J Epidemiol Community Health 2009; 63:50-5.

13. Barbosa-Branco A. O impacto do NTEP na caracterização dos acidentes de trabalho no Brasil. Revista CIPA 2008; 345:50-55.

14. Food Empowerment Project. Slaughterhouse workers. http://www.foodispower.org/slaughterhouse_workers.php (acessado em 10/Fev/2012).

15. Albuquerque PP, Settineri FS. Doenças do trabalho nos frigoríficos de carne: omissão e transferência de responsabilidade. Pelotas: CNTA-SUL, 2011. http://www.sticap.org.br/downloads/outros/Teias.pdf (acessado em 20/Jul/2011).

16. Roto P, Kivi P. Prevalence of epicondylitis and tenosynovitis among meatcutters. Scand J Work Environ Health 1984; 10:203-5.

17. Elders LA, Heinrich J, Burdorf A. Risk factors for sickness absence because of low back pain among scaffolders: a 3-year follow-up study. Spine (Phila Pa 1976) 2003; 28:1340-6.

18. Rios AO, Rego RCF, Pena PGL. Doenças em trabalhadores da pesca. Rev Baiana Saúde Pública 2011; 35:175-88.

19. Gluck JV, Oleinick A. Claim rates of compensable back injuries by age, gender, occupation, and industry. Do they relate to return-to-work experience? Spine (Phila Pa 1976) 1998; 23:1572-87.

20. Islam SS, Velilla AM, Doyle EJ, Ducatman AM. Gender differences in work-related injury/illness: analysis of workers compensation claims. Am J Ind Med 2001; 39:84-91.

21. Lindqvist K, Schelp L, Timpka T. Gender aspects of work-related injuries in a Swedish municipality. Safety Science 1999; 31:183-96.

22. Bekkera MHJ, Rutteb CG, Rijswijkc KV. Sickness absence: a gender-focused review. Psychol Health Med 2009; 14:405-18.

23. Silverstein B, Welp E, Nelson N, Kalat J. Claims incidence of work-related disorders of the upper extremities: Washington state, 1987 through 1995. Am J Public Health 1998; 88:1827-33.
24. Roquelaure Y, Ha C, Leclerc A, Touranchet A, Sauteron M, Melchior M, et al. Epidemiologic surveillance of upper-extremity musculoskeletal disorders in the working population. Arthritis Rheum 2006; 55:765-78.

25. Fitzgerald AJ, Kalof L, Dietz T. Slaughterhouses and increased crime rates: an empirical analysis of spillover from 'The Jungle' into the surrounding community. Organization and Environment 2009; 22:158-84.

26. Andersen JH, Kaergaard A, Mikkelsen S, Jensen UF, Frost P, Bonde JP, et al. Risk factors in the onset of neck/shoulder pain in a prospective study of workers in industrial and service companies. Occup Environ Med 2003;60:649-54.

27. Nelson DI, Concha-Barrientos M, Driscoll T, Steenland K, Fingerhut M, et al. The Global burden of selected occupational diseases and injury risks: methodology and summary. Am J Ind Med 2005; 48:400-18.

28. Allan J, Alston M, Dowling J, Ball P, Clifford A. Alcohol and drug use amongst fishing and farming workers: preliminary indications, perceptions and implications. In: 11th National Rural Health Conference; http://nrha.org.au/11nrhc/ papers/11th\%20NRHC\%20Allan_Julaine_C7.pdf (acessado em 04/Abr/2013).

29. Corrêa MJM. A construção social do silêncio epidemiológico do benzenismo: uma história negada [Dissertação de Mestrado]. Porto Alegre: Pontifícia Universidade Católica do Rio Grande do Sul; 2008.

30. Instituto Nacional de Câncer. Diretrizes para a vigilância do câncer relacionado ao trabalho. Rio de Janeiro: Instituto Nacional de Câncer; 2012.

31. Kines P, Hannerz H, Mikkelsen KL, Tüchsen F. Industrial sectors with high risk of women's hospitaltreated injuries. Am J Ind Med 2007;50:13-21.

32. Lipton B, Porter K, Nelson G. Workers compensation temporary total disability indemnity benefit duration. https://www.ncci.com/nccimain/ industryinformation/researchoutlook/pages/wctemp-benefit-2012-upate.aspx (acessado em 07/ Dez/2012).

33. Laaksonen M, Martikainen P, Rahkonen O, Lahelma E. Explanations for gender differences in sickness absence: evidence from middle-aged municipal employees from Finland. Occup Environ Med 2008; 65:325-30.

34. Souza NSS, Santana VS. Fatores associados à duração dos benefícios por incapacidade: um estudo de coorte. Rev Saúde Pública 2012; 46:425-34.

35. Cheadle A, Franklin G, Wolfhagen C, Savarino J, Liu PY, Salley C, et al. Factors influencing the duration of work-related disability: a population-based study of Washington State workers' compensation. Am J Public Health 1994;84:190e6.

Recebido em 17/Abr/2013

Versão final reapresentado em 29/Jul/2014

Aprovado em 08/Ago/2014 